Research Article

\title{
Some explicit expressions for twisted $q$-analogues of Catalan numbers and polynomials
}

\author{
Dongkyu Lim*
}

Department of Mathematics Education, Andong National University, Andong 36729, Republic of Korea

(Received: 8 January 2022. Received in revised form: 22 January 2022. Accepted: 25 January 2022. Published online: 28 January 2022.)

(c) 2022 the author. This is an open access article under the CC BY (International 4.0) license (www.creativecommons.org/licenses/by/4.0/).

\section{Abstract}

In this paper, the author considers the twisted $q$-analogues of Catalan numbers, which are arisen from the fermionic $p$-adic $q$-integrals. By using the fermionic $p$-adic $q$-integrals or generating functions, some explicit identities and properties for the twisted $q$-analogues of Catalan numbers and polynomials are given.

Keywords: twisted Catalan numbers; twisted $q$-analogues of Catalan numbers; fermionic $p$-adic $q$-integrals; Euler numbers; Stirling numbers.

2020 Mathematics Subject Classification: 05A19, 11B83.

\section{Introduction}

Although the Catalan numbers were named after the French-Belgian mathematician Eugène Charles Catalan (18141894), they were initially introduced by the Mongolian mathematician Ming Antu in 1730. The Catalan numbers $C_{n}$ are given $[7,15]$ by the generating function

$$
\sum_{n=0}^{\infty} C_{n} t^{n}=\sum_{n=0}^{\infty} \frac{1}{n+1}\left(\begin{array}{c}
2 n \\
n
\end{array}\right) t^{n}
$$

We note that

$$
\sqrt{1-4 t}=1-2 \sum_{m=0}^{\infty}\left(\begin{array}{c}
2 m \\
m
\end{array}\right) \frac{1}{m+1} t^{m+1}=1-2 \sum_{m=0}^{\infty} C_{m} t^{m+1}, \quad(0<|4 t|<1) .
$$

The Catalan numbers satisfy the recurrence relations

$$
C_{0}=1, \quad C_{n}=\sum_{m=0}^{n-1} C_{m} C_{n-1-m}, \quad(n \geq 1) .
$$

Also, the Catalan numbers form a sequence of positive integers

$$
1,1,2,5,14,42,132,429,1430,4862,16796,58786,208012, \cdots
$$

which is asymptotic to $4^{n} / n^{\frac{3}{2}} \sqrt{\pi}$, as $n$ tends to $\infty$, and appears in various counting problems. They count certain types of lattice paths, permutations, binary trees, and many other combinatorial objects. For more information on the Catalan numbers, please refer to $[5,7,9,11,12,15]$ and the closely related references therein.

Let $p$ be a fixed odd prime number. Throughout this paper, $\mathbb{Z}_{p}, \mathbb{Q}_{p}$ and $\mathbb{C}_{p}$ denote the ring of $p$-adic integers, the field of $p$-adic rational numbers and the completion of the algebraic closure of $\mathbb{Q}_{p}$, respectively. The $p$-adic norm $|\cdot|_{p}$ is normalized as $|p|_{p}=\frac{1}{p}$. The notation $[x]_{q}$ denotes

$$
[x]_{q}=\frac{1-q^{x}}{1-q} .
$$

Let $f(x)$ be a continuous $\mathbb{C}_{p}$-valued function on $\mathbb{Z}_{p}$. Then the fermionic $p$-adic integral on $\mathbb{Z}_{p}$ is defined [3] by the limit, if it exists,

$$
I_{-1}(f)=\int_{\mathbb{Z}_{p}} f(x) d \mu_{-1}(x)=\lim _{N \rightarrow \infty} \sum_{x=0}^{p^{N}-1} f(x)(-1)^{x} .
$$

From the above definition, we note that

$$
I_{-1}\left(f_{1}\right)=-I_{-1}(f)+2 f(0) \text {, where } f_{1}(x)=f(x+1) .
$$


Remarkably, it follows from [2] that the Catalan numbers are represented by the fermionic $p$-adic integral on $\mathbb{Z}_{p}$

$$
\begin{aligned}
\int_{\mathbb{Z}_{p}}(1-4 t)^{\frac{x}{2}} d \mu_{-1}(x) & =\frac{2}{1+\sqrt{1-4 t}} \\
& =\sum_{n=0}^{\infty} \frac{1}{n+1}\left(\begin{array}{c}
2 n \\
n
\end{array}\right) t^{n}=\sum_{n=0}^{\infty} C_{n} t^{n}, \quad(0<|4 t|<1) .
\end{aligned}
$$

where $t \in \mathbb{C}_{p}$ with $|t|_{p}<p^{-\frac{1}{p-1}}$. The application of the fermionic $p$-adic integral on $\mathbb{Z}_{p}$ is an effective way to deduce many important results for $q$-numbers and polynomials. For more information, please refer to [1,3,4,6,8,13,14].

Let $q$ be an indeterminate in $\mathbb{C}_{p}$ with $|1-q|_{p}<1$. It is known that the fermionic $p$-adic $q$-integral on $\mathbb{Z}_{p}$ is defined [4] by

$$
\begin{aligned}
I_{-q}(f) & =\int_{\mathbb{Z}_{p}} f(x) d \mu_{-q}(x)=\lim _{N \rightarrow \infty} \sum_{x=0}^{p^{N}-1} f(x) \mu_{-q}\left(x+p^{N} \mathbb{Z}_{p}\right) \\
& =\lim _{N \rightarrow \infty} \frac{1}{\left[p^{N}\right]_{-q}} \sum_{x=0}^{p^{N}-1} f(x)(-q)^{x},
\end{aligned}
$$

where $f$ is any continuous function on $\mathbb{Z}_{p}$. From the above definition, we can derive the following integral identity

$$
q I_{-q}\left(f_{1}\right)+I_{-q}(f)=[2]_{q} f(0) .
$$

The following are well known for the Stirling numbers of the first kind $S_{1}(n, k)$ for $n \geq 0$ can be generated by

$$
(x)_{n}=\sum_{k=0}^{n} S_{1}(n, k) x^{k} \quad \text { and } \quad \frac{1}{k !}(\log (1+t))^{k}=\sum_{n=k}^{\infty} S_{1}(n, k) \frac{t^{n}}{n !}
$$

where $(x)_{0}=1,(x)_{n}=x(x-1) \cdots(x-n+1),(n \geq 1)$. Further, for $n \geq 0$, the Stirling numbers of the second kind $S_{2}(n, k)$ are given by

$$
x^{n}=\sum_{k=0}^{n} S_{2}(n, k)(x)_{k}, \quad \frac{1}{k !}\left(e^{t}-1\right)^{k}=\sum_{n=k}^{\infty} S_{2}(n, k) \frac{t^{n}}{n !} .
$$

For $n \in \mathbb{N}$, let $T_{p}$ be the $p$-adic locally constant space defined by

$$
T_{p}=\bigcup_{n \geq 1} C_{p^{n}}=\lim _{n \rightarrow \infty} C_{p^{n}}
$$

where $C_{p^{n}}=\left\{w \mid w^{p^{n}}=1\right\}$ is the cyclic group of order $p^{n}$ (see $[6,10,13]$ ).

The aim of this paper is to construct a new type of numbers, the twisted $q$-analogues of Catalan numbers $C_{n, q, w}$ by virtue of a fermionic $p$-adic $q$-integral of $\mathbb{Z}_{p}$, and to investigate some properties and identities of these numbers.

\section{Twisted $q$-analogues of Catalan numbers}

Assume that $t, q \in \mathbb{C}_{p}$ such that

$$
|t|_{p}<p^{-\frac{1}{p-1}} \text { and }|q-1|_{p}<1 .
$$

For $w \in T_{p}$, let us take $f(x)=w^{x}(1-4 t)^{\frac{x}{2}}$ in (2). Then we have

$$
\int_{\mathbb{Z}_{p}} w^{x}(1-4 t)^{\frac{x}{2}} d \mu_{-q}(x)=\frac{[2]_{q}}{w q \sqrt{1-4 t}+1}=\frac{[2]_{q}}{1-w^{2} q^{2}+4 w^{2} q^{2} t}(1-w q \sqrt{1-4 t}), \quad(w \neq 1) .
$$

Motivated from (3), we consider the twisted $q$-analogues of Catalan numbers $C_{n, q, w}$ which are given by the generating function to be

$$
\frac{[2]_{q}}{1-w^{2} q^{2}+4 w^{2} q^{2} t}(1-w q \sqrt{1-4 t})=\sum_{n=0}^{\infty} C_{n, q, w} t^{n}, \quad(w \neq 1) .
$$

When $w=1$, we can recover the $q$-analogues of Catalan numbers, denoted by $C_{n, q}$, which are generated [5] by

$$
\frac{[2]_{q}}{1-2 q^{2}+4 q^{2} t}(1-q \sqrt{1-4 t})=\sum_{n=0}^{\infty} C_{n, q} t^{n}
$$

Especially $q \rightarrow 1$,

$$
\lim _{q \rightarrow 1} C_{n, q}=\frac{1}{2 t}(1-\sqrt{1-4 t})=\sum_{n=0}^{\infty} C_{n} t^{n}
$$


which recovers the Catalan numbers in (1). From (3) or (4), we observe that

$$
\begin{aligned}
\frac{[2]_{q}}{1-w^{2} q^{2}+4 w^{2} q^{2} t} & (1-w q \sqrt{1-4 t})=\frac{[2]_{q}}{1-w^{2} q^{2}+4 w^{2} q^{2} t}\left(1+w q \sum_{l=0}^{\infty}\left(\begin{array}{c}
2 l \\
l
\end{array}\right) \frac{1}{2 l-1} t^{l}\right) \\
& =\frac{[2]_{q}}{1-w^{2} q^{2}}\left(\frac{1}{1+\frac{4 w^{2} q^{2}}{1-w^{2} q^{2}}}\right)\left(1+w q \sum_{l=0}^{\infty}\left(\begin{array}{c}
2 l \\
l
\end{array}\right) \frac{1}{2 l-1} t^{l}\right) \\
& =\frac{[2]_{q}}{1-w^{2} q^{2}}\left(\sum_{m=0}^{\infty}\left(\frac{4 w^{2} q^{2}}{1-w^{2} q^{2}}\right)^{m}(-1)^{m} t^{m}\right)\left(1+w q \sum_{l=0}^{\infty}\left(\begin{array}{c}
2 l \\
l
\end{array}\right) \frac{1}{2 l-1} t^{l}\right) \\
& =\frac{[2]_{q}}{1-w^{2} q^{2}} \sum_{m=0}^{\infty}\left(\frac{4 w^{2} q^{2}}{1-w^{2} q^{2}}\right)^{m}(-1)^{m} t^{m}+\frac{[2]_{q} w q}{1-w^{2} q^{2}} \sum_{n=0}^{\infty} \sum_{l=0}^{n}\left(\begin{array}{c}
2 l \\
l
\end{array}\right) \frac{(-1)^{n-l}}{2 l-1}\left(\frac{4 w^{2} q^{2}}{1-w^{2} q^{2}}\right)^{n-l} t^{n} \\
& =\sum_{n=0}^{\infty}\left[\frac{[2]_{q}}{1-w^{2} q^{2}}\left\{\left(-\frac{4 w^{2} q^{2}}{1-w^{2} q^{2}}\right)^{n}+w q \sum_{l=0}^{n}\left(\begin{array}{c}
2 l \\
l
\end{array}\right) \frac{(-1)^{n-l}}{2 l-1}\left(\frac{4 w^{2} q^{2}}{1-w^{2} q^{2}}\right)^{n-l}\right\}\right] t^{n} .
\end{aligned}
$$

Combining this with (4) leads to the identity given in the next theorem.

Theorem 2.1. For $w \in T_{p}$ and $n \geq 0$, we have

$$
\begin{aligned}
C_{n, q, w} & =\frac{[2]_{q}}{1-w^{2} q^{2}}\left\{\left(-\frac{4 w^{2} q^{2}}{1-w^{2} q^{2}}\right)^{n}+w q \sum_{l=0}^{\infty}\left(\begin{array}{c}
2 l \\
l
\end{array}\right) \frac{(-1)^{n-l}}{2 l-1}\left(\frac{4 w^{2} q^{2}}{1-w^{2} q^{2}}\right)^{n-l}\right\} \\
& =\frac{[2]_{q} w q}{1-w^{2} q^{2}} \sum_{l=1}^{n}\left(\begin{array}{c}
2 l \\
l
\end{array}\right) \frac{(-1)^{n-l}}{2 l-1}\left(\frac{4 w^{2} q^{2}}{1-w^{2} q^{2}}\right)^{n-l}+\frac{[2]_{q}}{1+w q}\left(-\frac{4 w^{2}}{1-w^{2}}\right)^{n} .
\end{aligned}
$$

For example,

$$
\begin{aligned}
& C_{0, q, w}=\frac{[2]_{q}}{1+w q}, \quad C_{1, q, w}=\frac{2[2]_{q} w q}{(1+w q)^{2}}, \quad C_{2, q, w}=\frac{[2]_{q}\left(6 w^{2} q^{3}+2 w q\right)}{(1+w q)^{3}} \\
& C_{3, q, w}=\frac{[2]_{q}\left(20 w^{3} q^{5}+16 w^{2} q^{3}+4 w q\right)}{(1+w q)^{4}}, \quad C_{4, q, w}=\frac{[2]_{q}\left(70 w^{4} q^{7}+94 w^{3} q^{5}+50 w^{2} q^{3}+10 w q\right)}{(1+w q)^{5}}, \quad \ldots
\end{aligned}
$$

For the case $w=1$ in (5), we have

$$
C_{0, q}=1, \quad C_{1, q}=\frac{2 q}{1+q}, \quad C_{2, q}=\frac{6 q^{3}+2 q}{(1+q)^{2}}, \quad C_{3, q}=\frac{20 q^{5}+16 q^{3}+4 q}{(1+q)^{3}}, \quad C_{4, q}=\frac{70 q^{7}+94 q^{5}+50 q^{3}+10 q}{(1+w q)^{4}}, \quad \cdots,
$$

and $q \rightarrow 1$ in (5), we can derive the twisted Catalan numbers in [10]

$$
\begin{aligned}
C_{0, w} & =\frac{2}{1+w}, \quad C_{1, w}=\frac{4 w}{(1+w)^{2}}, \quad C_{2, w}=\frac{12 w^{2}+4 w}{(1+w)^{3}} \\
C_{3, w} & =\frac{8\left(5 w^{3}+4 w^{2}+w\right)}{(1+w)^{4}}, \quad C_{4, w}=\frac{70 w^{4}+94 w^{3}+50 w^{2}+10 w}{(1+w)^{5}}, \quad \cdots .
\end{aligned}
$$

Note that

$$
\begin{aligned}
& \lim _{q \rightarrow 1} \lim _{w \rightarrow 1} C_{0, q, w}=1=C_{0}, \quad \lim _{q \rightarrow 1} \lim _{w \rightarrow 1} C_{1, q, w}=1=C_{1}, \quad \lim _{q \rightarrow 1} \lim _{w \rightarrow 1} C_{2, q, w}=2=C_{2}, \\
& \lim _{q \rightarrow 1} \lim _{w \rightarrow 1} C_{3, q, w}=5=C_{3}, \quad \lim _{q \rightarrow 1} \lim _{w \rightarrow 1} C_{4, q, w}=14=C_{4},
\end{aligned}
$$$$
\lim _{q \rightarrow 1} \lim _{w \rightarrow 1} C_{n, q, w}=\lim _{w \rightarrow 1} \frac{[2]_{q}}{1-w q}\left\{\left(-\frac{4 w^{2} q^{2}}{1-w^{2} q^{2}}\right)^{n}+w q \sum_{l=0}^{\infty}\left(\begin{array}{c}
2 l \\
l
\end{array}\right) \frac{(-1)^{n-l}}{2 l-1}\left(\frac{4 w^{2} q^{2}}{1-w^{2} q^{2}}\right)^{n-l}\right\}=\left(\begin{array}{c}
2 n \\
n
\end{array}\right) \frac{1}{n+1}=C_{n}, \quad(n \geq 0) .
$$

\section{Twisted $q$-analogues of Catalan polynomials}

In this section, we assume that $t, q \in \mathbb{C}_{p}$ with $|t|_{p}<p^{-\frac{1}{p-1}}$ and $|q-1|_{p}<1$. For $w \in T_{p}$, we define the twisted $q$-analogues of Catalan polynomials $C_{n, q, w}(x)$ by the fermionic $p$-adic $q$-integral on $\mathbb{Z}_{p}$.

$$
\int_{\mathbb{Z}_{p}} w^{y}(1-4 t)^{\frac{x+y}{2}} d \mu_{-q}(y)=\frac{[2]_{q}}{w q \sqrt{1-4 t}+1}(1-4 t)^{\frac{x}{2}}=\sum_{n=0}^{\infty} C_{n, q, w}(x) t^{n} .
$$


When $x=0, C_{n, q, w}=C_{n, q, w}(0)$ are the twisted $q$-analogue Catalan numbers in (3).

Now, we want to give relations between the twisted $q$-analogues of Catalan polynomials and the twisted $q$-Euler polynomials. Recall from [6] that the twisted $q$-Euler polynomials $E_{n, q, w}(x)$ are generated by

$$
\sum_{n=0}^{\infty} E_{n, q, w}(x) \frac{t^{n}}{n !}=\frac{[2]_{q}}{w q e^{t}+1} e^{t x}=\int_{\mathbb{Z}_{p}} w^{y} e^{(x+y) t} d \mu_{-q}(y) .
$$

When $x=0, E_{n, q, w}=E_{n, q, w}(0)$ are the twisted $q$-Euler numbers.

For $w \in T_{p}$, by (6) and (7), it follows that

$$
\begin{aligned}
\sum_{n=0}^{\infty} C_{n, q, w}(x) t^{n} & =\int_{\mathbb{Z}_{p}} w^{y}(1-4 t)^{\frac{x+y}{2}} d \mu_{-q}(y) \\
& =\int_{\mathbb{Z}_{p}} w^{y} \exp \left(\left(\frac{x+y}{2}\right) \log (1-4 t)\right) d \mu_{-q}(y) \\
& =\sum_{m=0}^{\infty} \frac{1}{2^{m}} m !(\log (1-4 t))^{m} \int_{\mathbb{Z}_{p}} w^{y}(x+y)^{m} d \mu_{-q}(y) \\
& =\sum_{m=0}^{\infty} 2^{-m} E_{m, q, w}(x) \sum_{n=m}^{\infty} S_{1}(n, m) \frac{(-4 t)^{n}}{n !} \\
& =\sum_{n=0}^{\infty}\left(\sum_{m=0}^{n} 2^{-m} E_{m, q, w}(x) S_{1}(n, m) \frac{(-4)^{n}}{n !}\right) t^{n} .
\end{aligned}
$$

Equating coefficients of $t^{n}$ gives the next result.

Theorem 3.1. For $w \in T_{p}$ and $n \geq 0$, we have

$$
C_{n, q, w}(x)=\frac{(-1)^{n}}{n !} \sum_{m=0}^{n} 2^{2 n-m} E_{m, q, w}(x) S_{1}(n, m) .
$$

Replacing $t$ with $\frac{1}{4}\left(1-e^{2 t}\right)$ in (6) yields

$$
\begin{aligned}
\sum_{m=0}^{\infty} E_{m, q, w}(x) \frac{t^{m}}{m !} & =\int_{\mathbb{Z}_{p}} w^{y} e^{(x+y) t} d \mu_{-q}(y) \\
& =\sum_{n=0}^{\infty} \frac{(-1)^{n} C_{n, q, w}(x)}{4^{n}}\left(e^{2 t}-1\right)^{n} \\
& =\sum_{n=0}^{\infty} \frac{(-1)^{n} C_{n, q, w}(x)}{4^{n}} \sum_{m=n}^{\infty} S_{2}(m, n) \frac{2^{m} t^{m}}{m !} \\
& =\sum_{m=0}^{n}\left(\sum_{n=0}^{m}(-1)^{n} 2^{m-2 n} n ! S_{2}(m, n) C_{n, q, w}(x)\right) \frac{t^{m}}{m !} .
\end{aligned}
$$

Therefore, we obtain the inversion formula of Theorem 3.1.

Theorem 3.2. For $w \in T_{p}$ and $m \geq 0$, we have

$$
E_{m, q, w}(x)=\sum_{n=0}^{m}(-1)^{n} 2^{m-2 n} n ! S_{2}(m, n) C_{n, q, w}(x) .
$$

Alternatively, $C_{n, q, w}(x)$ can be expressed as follows:

$$
\begin{aligned}
\sum_{n=0}^{\infty} C_{n, q, w}(x) t^{n} & =\int_{\mathbb{Z}_{p}} w^{y}(1-4 t)^{\frac{x+y}{2}} d \mu_{-q}(y) \\
& =\sum_{n=0}^{\infty}(-4)^{n} \int_{\mathbb{Z}_{p}} w^{y}\left(\frac{x+y}{2}\right)_{n} d \mu_{-q}(y) \frac{t^{n}}{n !}=\sum_{n=0}^{\infty}(-4)^{n} \int_{\mathbb{Z}_{p}} w^{y}\left(\begin{array}{c}
\frac{x+y}{2} \\
n
\end{array}\right) d \mu_{-q}(y) t^{n} .
\end{aligned}
$$

Comparing this with (8) leads to the the identity given in the next theorem. 
Theorem 3.3. For $w \in T_{p}$ and $n \geq 0$, we have

$$
\int_{\mathbb{Z}_{p}} w^{y}\left(\begin{array}{c}
\frac{x+y}{2} \\
n
\end{array}\right) d \mu_{-q}(y)=(-1)^{n} \frac{C_{n, q, w}(x)}{4^{n}}, \quad\left(x, y \in \mathbb{Z}_{p}\right) .
$$

For $\lambda \in \mathbb{Z}_{p},|t|_{p}<p^{-\frac{1}{p-1}}$ and $w \in T_{p}$, the twisted $(q, \lambda)$-Changhee polynomials $C h_{n, q, w}(x \mid \lambda)$ are defined by the generating function

$$
\frac{[2]_{q}}{w q(1+t)^{\lambda}+1}(1+t)^{x}=\sum_{n=0}^{\infty} C h_{n, q, w}(x \mid \lambda) \frac{t^{n}}{n !} .
$$

Thus by (2), we get the fermionic $p$-adic $q$-integral representation of $C h_{n, q, w}(x \mid \lambda)$ by

$$
\int_{\mathbb{Z}_{p}} w^{y}(1+t)^{\lambda y+x} d \mu_{-q}(y)=\frac{[2]_{q}}{w q(1+t)^{\lambda}+1}(1+t)^{x}=\sum_{n=0}^{\infty} C h_{n, q, w}(x \mid \lambda) \frac{t^{n}}{n !}, \quad\left(x \in \mathbb{Z}_{p}\right) .
$$

When $x=0$, we obtain the twisted $(q, \lambda)$-Changhee numbers $C h_{n, q, w}(\lambda)=C h_{n, q, w}(0 \mid \lambda)$, which are generated by

$$
\frac{[2]_{q}}{w q(1+t)^{\lambda}+1}=\sum_{n=0}^{\infty} C h_{n, q, w}(\lambda) \frac{t^{n}}{n !}=\int_{\mathbb{Z}_{p}} w^{y}(1+t)^{\lambda y} d \mu_{-q}(y) .
$$

We note that $C h_{0, q, w}(\lambda)=\frac{1+q}{1+w q}$. Specially $w=1, C h_{n, q}(\lambda)=C h_{n, q, 1}(\lambda)$ is the $n$th $(\lambda, q)$-Changhee numbers in [5]. Hence we have from (6) and (10)

$$
\sum_{n=0}^{\infty} C_{n, q, w} t^{n}=\frac{[2]_{q}}{w q \sqrt{1-4 t}+1}=\sum_{n=0}^{\infty} C h_{n, q, w}\left(\frac{1}{2}\right)(-4)^{n} \frac{t^{n}}{n !}
$$

Futhermore, by using (6) and (9), we obtain

$$
\sum_{n=0}^{\infty} C_{n, q, w}(x) t^{n}=\int_{\mathbb{Z}_{p}} w^{y}(1-4 t)^{\frac{x+y}{2}} d \mu_{-q}(y)=\sum_{n=0}^{\infty} C h_{n, q, w}\left(\frac{x}{2} \mid \frac{1}{2}\right)(-4)^{n} \frac{t^{n}}{n !} .
$$

Comparing the coefficients on both sides of (11) and (12) respectively, we obtain the next result.

Theorem 3.4. For $w \in T_{p}$ and $n \geq 0$, we have

(i) $n ! C_{n, q, w}=(-4)^{n} C h_{n, q, w}\left(\frac{1}{2}\right)$,

(ii) $n ! C_{n, q, w}(x)=(-4)^{n} C h_{n, q, w}\left(\frac{x}{2} \mid \frac{1}{2}\right)$.

It is easy to see that the generating function of $C_{n, q, w}(x)$ can be reformulated as

$$
\begin{aligned}
\sum_{n=0}^{\infty} C_{n, q, w}(x) t^{n} & =\frac{[2]_{q}}{w q \sqrt{1-4 t}+1}(1-4 t)^{\frac{x}{2}} \\
& =\left(\sum_{l=0}^{\infty} C_{l, q, w} t^{l}\right)\left(\sum_{m=0}^{\infty}\left(\begin{array}{c}
\frac{x}{2} \\
m
\end{array}\right)(-4)^{m} t^{m}\right)=\sum_{n=0}^{\infty}\left(\sum_{m=0}^{n}\left(\begin{array}{c}
\frac{x}{2} \\
m
\end{array}\right)(-4)^{m} C_{n-m, q, w}\right) t^{n} .
\end{aligned}
$$

This implies another expression for the twisted $q$-analogues of Catalan polynomials

$$
C_{n, q, w}(x)=\sum_{m=0}^{n}\left(\begin{array}{c}
\frac{x}{2} \\
m
\end{array}\right)(-4)^{m} C_{n-m, q, w}
$$

Whereas we observe that

$$
\begin{aligned}
(1-4 t)^{\frac{x}{2}} & =\sum_{l=0}^{\infty}\left(\frac{x}{2}\right)^{l} \frac{1}{l !}(\log (1-4 t))^{l} \\
& =\sum_{l=0}^{\infty}\left(\frac{x}{2}\right)^{l} \sum_{m=l}^{\infty} S_{1}(m, l)(-4)^{m} \frac{t^{m}}{m !}=\sum_{m=0}^{\infty}\left(\sum_{l=0}^{m} S_{1}(m, l) \frac{(-4)^{m}}{m !}\left(\frac{x}{2}\right)^{l}\right) t^{m} .
\end{aligned}
$$

Combination of this identity with (13) results in

$$
\begin{aligned}
\sum_{n=0}^{\infty} C_{n, q, w}(x) t^{n}=\frac{[2]_{q}}{w q \sqrt{1-4 t}+1}(1-4 t)^{\frac{x}{2}} & =\left(\sum_{k=0}^{\infty} C_{k, q, w} t^{k}\right)\left(\sum_{m=0}^{\infty}\left(\sum_{l=0}^{m} S_{1}(m, l) \frac{(-4)^{m}}{m !}\left(\frac{x}{2}\right)^{l}\right) t^{m}\right) \\
& =\sum_{n=0}^{\infty}\left(\sum_{m=0}^{n} \sum_{l=0}^{m} S_{1}(m, l) \frac{(-4)^{m}}{m !}\left(\frac{x}{2}\right)^{l} C_{n-m, q, w}\right) t^{n} .
\end{aligned}
$$


Equating coefficients on the very ends of the above identity arrives at another expression for $C_{n, q, w}(x)$.

$$
C_{n, q, w}(x)=\sum_{m=0}^{n} \sum_{l=0}^{m} S_{1}(m, l) \frac{(-4)^{m}}{m !}\left(\frac{x}{2}\right)^{l} C_{n-m, q, w} .
$$

Therefore, by (13) and (14), we have the next result.

Theorem 3.5. For $w \in T_{p}$ and $n \geq 0$, we have

$$
C_{n, q, w}(x)=\sum_{m=0}^{n}\left(\begin{array}{c}
\frac{x}{2} \\
m
\end{array}\right)(-4)^{m} C_{n-m, q, w}=\sum_{m=0}^{n} \sum_{l=0}^{m} S_{1}(m, l) \frac{(-4)^{m}}{m !}\left(\frac{x}{2}\right)^{l} C_{n-m, q, w} .
$$

Remark 3.1. In view of Theorems 3.1-3.5, by specializing $q \rightarrow 1$, we can obtain several interesting identities for the twisted Catalan polynomials $C_{n, w}(x)$.

Remark 3.2. In view of Theorems 3.1-3.5, by specializing $w=1$, we can recover the identities for the q-analogues of Catalan polynomials $C_{n, q}(x)$ in [5].

\section{Conclusion}

In this paper, we introduced the twisted $q$-analogues of the Catalan numbers $C_{n, q, w}$ with the help of a fermionic $p$-adic $q$-integral of $\mathbb{Z}_{p}$ and derive explicit expressions and some identities for those numbers. In particular, we deduced explicit expressions of $C_{n, q, w}$, as a rational function in $q$ and $w$, in terms of the twisted $q$-Euler numbers and Stirling numbers of the first kind, as a fermionic $p$-adic $q$-integral on $\mathbb{Z}_{p}$, and involving the twisted $(q, \lambda)$-Changhee numbers. In addition, we considered a polynomial extension of the twisted $q$-analogues of Catalan numbers, namely the twisted $q$-analogues of Catalan polynomials $C_{n, q, w}(x)$, and derived explicit expressions in terms of the twisted Catalan numbers and Stirling numbers of the first kind and of the twisted $q$-Euler polynomials and Stirling numbers of the first kind.

\section{Acknowledgment}

The author would like to thank the anonymous referees for their comments and suggestions which improved the original version of this paper. This work was partially supported by the National Research Foundation of Korea (NRF), grant funded by the Korean government (MSIT), NRF-2021R1C1C1010902.

\section{References}

[1] S. Araci, M. Acikgoz, E. Sen, On the extended Kim's $p$-adic $q$-deformed fermionic integrals in the $p$-adic integer ring, J. Number Theory 133 (2013) $3348-3361$.

[2] D. V. Dolgy, G.-W. Jang, D. S. Kim, T. Kim, Explicit expressions for Catalan-Daehee numbers, Proc. Jangjeon Math. Soc. 20 (2017) 1-9.

[3] T. Kim, $q$-Volkenborn integration, Russ. J. Math. Phys. 9 (2002) 288-299.

[4] T. Kim, A note on $p$-adic $q$-integral on $\mathbb{Z}_{p}$ associated with $q$-Euler numbers, Adv. Stud. Contemp. Math. 15 (2007) $133-137$.

[5] T. Kim, D. S. Kim, J. Kwon, A note on $q$-analogue of Catalan numbers arising from fermionic $p$-adic $q$-integral on $\mathbb{Z}_{p}$, Adv. Stud. Contemp. Math. 31 (2021) 279-286.

[6] T. Kim, S.-H. Rim, On the twisted $q$-Euler numbers and polynomials associated with basic $q$-l-functions, J. Math. Anal. Appl. 336 (2007) 738-744.

[7] T. Koshy, Catalan Numbers with Applications, Oxford University Press, Oxford, 2009.

[8] I. Kucukoglu, Y. Simsek, Construction and computation of unified Stirling-type numbers emerging from $p$-adic integrals and symmetric polynomials, Rev. R. Acad. Cienc. Exactas Fís. Nat. Ser. A Mat. 115 (2021) \#167.

[9] I. Kucukoglu, B. Simsek, Y. Simsek, New classes of Catalan-type numbers and polynomials with their applications related to $p$-adic integrals and computational algorithms, Turk. J. Math. 44 (2020) 2337-2355.

[10] D. Lim, Some explicit expressions for twisted Catalan-Daehee numbers, Symmetry 14 (2022) \#189.

[11] F. Qi, X.-T. Shi, F.-F. Liu, An integral representation, complete monotonicity, and inequalities of the Catalan numbers, Filomat 32 (2018) $575-587$.

[12] F. Qi, Q. Zou, B.-N. Guo, The inverse of a triangular matrix and several identities of the Catalan numbers, Appl. Anal. Discrete Math. 13 (2019) $518-541$.

[13] Y. Simsek, Twisted p-adic ( $h, q)$-L-functions, Comput. Math. Appl. 59 (2010) 2097-2110.

[14] Y. Simsek, Explicit formulas for $p$-adic integrals: Approach to $p$-adic distributions and some families of special numbers and polynomials, Montes Taurus J. Pure Appl. Math. 1 (2019) 1-76.

[15] R. P. Stanley, Catalan Numbers, Cambridge University Press, New York, 2015. 